\title{
Routine clinical variables predicted mortality in patients with heart failure and systolic dysfunction
}

Brophy JM, Dagenais GR, McSherry F, et al. A multivariate model for predicting mortality in patients with heart failure and systolic dysfunction. Am J Med 2004;116:300-4.

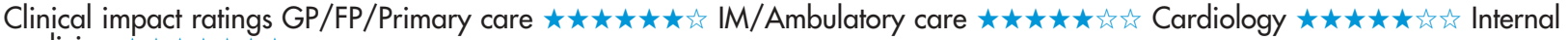

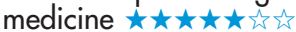

In patients with heart failure (HF) and depressed systolic function, can routine clinical variables be used to predict short term and long term mortality?

\section{METHODS}

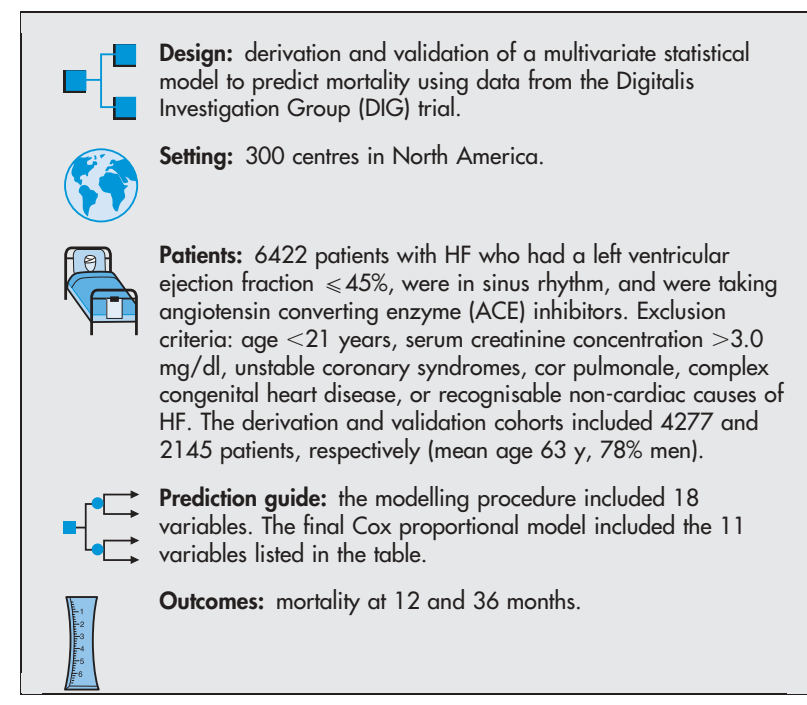

\section{MAIN RESULTS}

Total mortality in the derivation sample was $11 \%$ at 12 months and $30 \%$ at 36 months. Independent predictors are summarised in the table. In the lowest risk decile, 12 month predicted mortality was $4.0 \%$ compared with $3.8 \%$ observed mortality, and 36 month predicted mortality was $11.8 \%$ compared with $10.7 \%$. In general, the models performed less well for the highest risk decile.

For correspondence: Dr J Brophy, McGill University Health Center, Montréal, Québec, Canada. jbroph@po-box.mcgill.ca

Source of funding: not stated.

\section{CONCLUSION}

Routine clinical variables predicted 12 and 36 month mortality in patients with heart failure and systolic dysfunction who were receiving angiotensin converting enzyme inhibitors.

\section{Commentary}

B failu rophy et al have contributed a model that predicts the likelihood of mortality at 12 and 36 months in patients with congestive heart failure (CHF). The authors used rigorous methods, including a Bayesian approach intended to minimise the risk of over-fitting and optimise prediction in future patients with similar characteristics. Indeed, the calibration curves by decile of risk suggest good performance in the validation dataset.

The model variables do not require sophisticated laboratory or imaging studies, but the equation requires 11 variables (table), including a composite variable of 7 clinical signs and symptoms. Although a computer-based tool may enable calculation of a risk score, it is unclear whether these variables can be easily or reliably obtained in routine clinical practice. Importantly, it is unclear how or why busy clinicians would use such a complex prognostic tool.

The authors suggest that risk models may be useful when selecting treatments for patients with CHF. Now that several mortality reducing treatment options are available, risk stratification may have a role in targeting more intense therapies to those most likely to benefit. This will only become clear when such models are used to stratify patients within clinical trials testing these therapies. The critical test is whether such stratification enables identification of patients at such low risk that they are unlikely to benefit from additional therapies to an extent that justifies the risks and costs of therapy. However, in this sample that included NYHA Class I-IV patients who were all receiving ACE inhibitors, the average 3 year mortality rate was $30 \%$, and the mortality rate of even the lowest risk decile was about $10 \%$. This suggests that even patients at low and moderate risk have considerable room for improvement beyond therapy with ACE inhibitors.

David M Kent, MD, MS

Tufts-New England Medical Center, Boston, Massachusetts, USA

Independent predictors of mortality in heart failure and systolic dysfunction*

\begin{tabular}{llll}
\hline & & \multicolumn{2}{c}{ Hazard ratio (95\% confidence interval) } \\
\cline { 3 - 4 } Variable & Reference group & Mortality at 12 months & Mortality at 36 months \\
\hline Age & $<50$ years & Not significant & $1.20(1.13$ to 1.28$)$ \\
Ejection fraction (per $10 \%$ decrease) & Class I & $1.34(1.22$ to 1.48$)$ & $1.34(1.22$ to 1.48$)$ \\
NYHA class & & $1.44(1.24$ to 1.68$)$ & $1.29(1.18$ to 1.41$)$ \\
Cardiothoracic ratio $>50 \%$ & $0-1$ signs or symptoms & $1.60(1.29$ to 1.97$)$ & $1.34(1.19$ to 1.52$)$ \\
Clinical signs or symptomst & $1.21(1.06$ to 1.38$)$ & $1.14(1.06$ to 1.24$)$ \\
Serum creatinine (per $\mathrm{mg} / \mathrm{dl}$ increase) & $>29.7 \mathrm{~kg} / \mathrm{m}^{2}$ & $1.85(1.49$ to 2.28$)$ & $1.73(1.51$ to 2.02$)$ \\
Body mass index & $1.18(1.08$ to 1.28$)$ & $1.09(1.04$ to 1.15$)$ \\
Diastolic BP & $>80 \mathrm{~mm} \mathrm{Hg}$ & $1.17(1.08$ to 1.28$)$ & Not significant \\
Systolic BP & $>139 \mathrm{~mm} \mathrm{Hg}$ & Not significant & $1.11(1.06$ to 1.16$)$ \\
Nitrates & & Not significant & $1.18(1.06$ to 1.32$)$ \\
Diabetes ischaemic aetiology & $1.46(1.19$ to 1.79$)$ & $1.43(1.26$ to 1.63$)$
\end{tabular}

${ }^{\star B P}$ = blood pressure. Hazard ratios are per 1 group increase for the following categorical variables: age, New York Heart Association (NYHA) class, clinical signs or symptoms, and per 1 group decrease for body mass index and blood pressure.

†Rales, increased jugular venous pressure, peripheral oedema, dyspnoea, limitation of activity, $\mathrm{S}_{3}$ gallop, or radiological evidence of pulmonary congestion. 